\title{
Balkanologie
}

Balkanologie Revue d'études pluridisciplinaires

Vol. $16 n^{\circ} 1 \mid 2021$

Au-delà de la "route des Balkans ": mondes sociaux des circulations

\section{Introduction. Au-delà de la « route des Balkans » : mondes sociaux des circulations}

Introduction. Beyond "the Balkan Route": Social Worlds of Circulation

\section{Guillaume Javourez}

\section{(2) OpenEdition}

\section{Journals}

Édition électronique

URL : https://journals.openedition.org/balkanologie/3194

DOI : 10.4000/balkanologie.3194

ISSN : 1965-0582

Éditeur

Association française d'études sur les Balkans (Afebalk)

Référence électronique

Guillaume Javourez, «Introduction. Au-delà de la « route des Balkans »: mondes sociaux des circulations », Balkanologie [En ligne], Vol. 16 n 1 | 2021, mis en ligne le 01 juin 2021, consulté le 04 octobre 2021. URL : http://journals.openedition.org/balkanologie/3194 ; DOI : https://doi.org/10.4000/ balkanologie.3194

Ce document a été généré automatiquement le 4 octobre 2021.

(c) Tous droits réservés 


\section{Introduction. Au-delà de la « route des Balkans » : mondes sociaux des circulations}

Introduction. Beyond "the Balkan Route": Social Worlds of Circulation

Guillaume Javourez

$1 \mathrm{Au}$ cours de l'année 2015, la détérioration de la situation socio-économique et l'intensification des combats dans certains pays du Moyen-Orient ont entraîné d'importants flux de population ${ }^{1}$. Si une majorité a trouvé refuge dans les pays frontaliers (Turquie, Jordanie ou Liban en tête), ils sont plusieurs centaines de milliers à avoir cherché à rejoindre au sein de l'espace Schengen les pays d'Europe de l'Ouest et du Nord. Face au renforcement des contrôles en Méditerranée centrale et occidentale, cette vague s'est progressivement réorientée vers la péninsule balkanique ${ }^{2}$, mettant en pleine lumière une "route des Balkans » de plus en plus empruntée depuis le début des années $2010^{3}$. Mais ces mouvements migratoires qui traversent aujourd'hui la péninsule sont également dans la continuité des mobilités qui ont touché la Grèce à partir des années 2000 , lorsque la frontière gréco-turque est devenue un point chaud des routes migratoires à destination de l'Union européenne (UE) ${ }^{4}$. Dans ce contexte, la libéralisation par la Turquie du régime de visas appliqué à son voisinage a contribué à l'émergence d'Istanbul comme plateforme de transit pour les migrants venant tant d'Afrique $^{5}$ que d'Asie et du Moyen-Orient ${ }^{6}$ avant de se rendre en Grèce, dans l'espace Schengen, et éventuellement de tenter de poursuivre leur route vers d'autres États de l'UE. Alors que les pays de la région étaient auparavant principalement perçus comme des pays de départ, ils sont devenus durant la décennie 2010 des pays dits de «transit ${ }^{7}$ » le long d'un corridor qui a été un temps organisé, avant de se fermer progressivement entre la fin de l'année 2015 et le début de $2016^{8}$. Cet événement marque une rupture avec une longue histoire migratoire caractérisée par l'importance de l'émigration et des mobilités intra régionales.

Mettant en perspective cet épisode récent, le présent dossier s'attache à rendre compte de phénomènes de circulation qui touchent la péninsule balkanique en prenant en 
considération le temps long. Il s'appuie sur une vision de la circulation qui met l'accent sur les dimensions matérielles, immatérielles et symboliques des mobilités humaines dont elle est indissociable. Elle permet cependant de déplacer la focale sur ce qui se déplace et relie les différents espaces de la mobilité qui se retrouvent indirectement mis en contact. Au carrefour du local et du global, l'étude des circulations de personnes, de biens et d'idées conduit alors à interroger les reconfigurations des sociétés contemporaines à la lumière de processus globaux qui voient la confrontation entre structures stato-centrées (État-nations) et structures multi-centrées (entités supranationales, réseaux migratoires, diaspora, réseaux marchands, etc.). Inspirée de la dialectique entre circulation et iconographie pensée par le géographe Jean Gottmann", l'étude des circulations dans les Balkans revient alors à étudier les interactions permanentes entre des dynamiques qui, loin de s'opposer, se nourrissent parfois. Ce dossier s'interrogera donc sur l'impact des processus de circulation sur les sociétés des pays concernés, mais aussi, en retour, sur la façon dont ces processus ont pu être influencés, orientés, parfois forcés, par les discours et les représentations des acteurs engagés sur ces parcours.

\section{Les espaces-temps des circulations}

Dans les années 1990, ce sont d'autres phénomènes migratoires qui attirent l'attention de la recherche, notamment francophone. Dans le contexte particulier de l'exYougoslavie, les flux de réfugiés et de déplacés dominent alors l'actualitée ${ }^{10}$. Plus largement, suite à l'effondrement des régimes socialistes, la levée partielle des obstacles à la circulation a entraîné l'apparition de phénomènes nouveaux par leur ampleur plus que par leur nature ${ }^{11}$. Émigration, travail et échanges transfrontaliers, migrations pendulaires et commerce de valise sont autant de modalités qui caractérisent les mobilités en provenance des pays balkaniques anciennement socialistes durant la décennie 1990 et au début des années $2000^{12}$. Les effets de la mondialisation sur les phénomènes migratoires ont conduit à la diversification de leurs formes et espaces, et l'attention porte alors sur les différentes formes de la mobilité afin de prendre en compte l'ensemble des mouvements de population, et notamment ceux de courte durée ${ }^{13}$ qui se multipliaient.

4 Si cette thématique prospère au cours des années 2000, c'est parce que les modifications réglementaires imposées par l'UE aux pays candidats sur les questions migratoires sont venues bouleverser le fonctionnement qui avait émergé après 1991. Lancée dans le cadre d'une procédure d'intégration européenne plaçant le contrôle de la frontière extérieure au centre de ses préoccupations, la "sécurisation accélérée ${ }^{14}$ " de la frontière risquait de mettre à mal ces dynamiques spatiales, formes à part entière d'intégration régionale, via l'introduction d'un régime de visas concernant les ressortissants des pays d'Europe de l'Est et des pays balkaniques non appelés à intégrer l'Union en $2004^{15}$. Les travaux menés par Bénédicte Michalon en 2007 sur la frontière roumano-moldave illustrent la façon dont la construction de la frontière extérieure de l'UE a constitué une entrave importante aux relations transfrontalières, source principale de revenus pour un nombre significatif de Moldaves de la région ${ }^{16}$. De même, l'entrée de la Bulgarie dans l'Union s'est traduite par une baisse importante des mobilités en provenance de Macédoine du Nord ${ }^{17}$. Balkanologie y a consacré deux dossiers en 2003 et 2008 , le premier portant sur les migrations et diasporas issues des 
Balkans à partir des années 1990, le second sur les imaginaires et itinéraires migratoires bulgares en Europe ${ }^{18}$. L'attention portée à ces phénomènes a par la suite décru au fil de la décennie suivante, progressivement remplacée par les thématiques du contrôle migratoire et par une forme de "normalisation » des situations migratoires en Europe de l'Est et du Sud-Est, faisant suite à l'adhésion de plusieurs de ces pays à l'UE et à la levée progressive des régimes de visas de court séjour, ayant contribué à une augmentation massive des mobilités transfrontalières dans la région ${ }^{19}$.

$5 \quad$ Les grandes lignes présentées ci-dessus ne doivent pas masquer la grande diversité des situations entre les pays des Balkans. Si l'année 1990 marque une rupture conduisant à l'ouverture des pays dont les régimes politiques étaient des plus restrictifs au regard des mobilités internationales (Albanie en tête, Bulgarie et Roumanie), la période est très différente dans le cas de la Grèce et des États successeurs de la Yougoslavie. À la différence de leurs voisins, la Grèce et la Yougoslavie avaient connu une importante émigration de travailleurs ${ }^{20}$, après la Seconde Guerre mondiale pour la Grèce ${ }^{21}$ et à partir des années 1960 pour la Yougoslavie socialiste. Leurs trajectoires commencent cependant à diverger à partir des années 1970 et 1980, alors que le solde migratoire de la Grèce redevient positif suite aux nombreuses migrations de retour liées à l'amélioration $d u$ contexte politique et économique $d u$ pays $^{22}$. De son côté, la Yougoslavie enregistre également des retours et les mobilités décroissent fortement après 1973, mais le phénomène est plus subi, provoqué par la suspension des recrutements décidée par les pays d'Europe de l'Ouest ${ }^{23}$.

6 Le tournant des années 1990 n'en marque pas moins une rupture au regard de la position de ces pays dans le système migratoire international. Dans le cas grec, les années 1990 voient l'arrivée d'une première vague de forte immigration avec l'installation de nombreux citoyens albanais venus chercher une vie meilleure dans une République hellénique qui constituait pour eux une "Amérique par défaut ${ }^{24}$ ». Ces mobilités, initiées dès la chute du régime hérité d'Enver Hoxha, auraient alors concerné entre 300000 et 500000 personnes durant cette décennie ${ }^{25}$, contribuant à un bouleversement profond de la société grecque et faisant alors de l'Albanie le principal pays d'émigration d'Europe en proportion de sa population. Dans le même temps, la Grèce devenait également un pays d'accueil pour des travailleurs venus d'autres pays balkaniques ou d'Europe de l'Est, en particulier de Géorgie, d'Ukraine ${ }^{26}$, de Bulgarie et de Roumanie. Au début des années 2000, les étrangers originaires de pays des Balkans représentaient ainsi $65 \%$ du total des étrangers recensés dans le pays ${ }^{27}$.

7 L'émigration économique marque en revanche l'espace post-yougoslave au-delà des guerres et conflits qui ont traversé la région de 1991 à 2001. Le Kosovo, la BosnieHerzégovine ou encore la Macédoine du Nord connaissent une émigration importante vers des pays tels que l'Allemagne, l'Autriche, la Suisse, la Suède et le Danemark. Mais les mouvements de réfugiés et les déplacements de populations y constituent néanmoins l'élément le plus marquant de la décennie 1990. Environ un quart des habitants de Bosnie-Herzégovine auraient ainsi été réfugiés à l'étranger, principalement en Europe de l'Ouest. Nombre d'entre eux, réfugiés suite à une "protection temporaire", ont ensuite été "rapatriés" dans leur pays d'origine, rejoignant bien souvent la cohorte des déplacés que la redéfinition des cartes sur des bases ethno-nationales avait engendrée durant le conflit ${ }^{28}$. La Serbie recensait pour sa part, en 1996, 617728 réfugiés en provenance de Croatie et de Bosnie-Herzégovine, des 
réfugiés auxquels se sont ajoutés de nombreux déplacés venus du Kosovo à la suite de l'intervention de l'OTAN en 1999 et aux violentes émeutes de mars $2004^{29}$.

8 Ces phénomènes migratoires contemporains se déroulent dans une région où les circulations humaines ont occupé une place particulière au cours de l'histoire. Dans un espace longtemps sous domination ottomane, les mobilités le long des routes commerciales représentaient un rouage essentiel de l'organisation spatiale de l'Empire ${ }^{30}$. Prenant en compte ce temps long, les migrations de travail et l'insertion de citoyens de ces États dans les réseaux de "Gastarbeiter », travailleurs «invités », ne doivent pas être vues comme une innovation contemporaine. À la fin du xixe siècle, déjà, des sujets ottomans venus d'Albanie ou de la Macédoine d'alors embarquaient en nombre sur des navires en vue d'une émigration transatlantique ${ }^{31}$ souvent temporaire puis permanente, formant des réseaux de circulation denses comme ceux qui liaient la Macédoine, Toronto, Chicago ou Détroit ${ }^{32}$. Ces mobilités internationales s'inscrivent également dans le cadre plus large de la pečalba, parfois désignée par le terme de gurbet, migration de travail périodique qui concerne la Macédoine de la période ottomane jusqu'à la période yougoslave ${ }^{33}$. Fréquemment associés à des activités agricoles ou artisanales, les pečalbari quittaient le plus souvent des zones montagneuses de la péninsule balkanique en direction des plaines, des vallées ou des villes de la région ${ }^{34}$ et restent encore aujourd'hui une figure de référence des migrations balkaniques contemporaines ${ }^{35}$.

9 Autre phénomène marquant de la décennie 1990, les déplacements de réfugiés apparaissent également dans la région comme un trait régulier des mobilités régionales $\mathrm{au} \mathrm{xx}^{\mathrm{e}}$ siècle. Des guerres balkaniques à la guerre civile grecque, la fragmentation politique et sociale progressive de la péninsule a entraîné un grand nombre de déplacements contraints. À titre d'exemples, la "Convention relative à l'émigration volontaire et réciproque » entre la Grèce et la Bulgarie en 1919, les échanges forcés de population entre la Grèce et la Turquie suite aux accords de Lausanne en 1923, le départ des Çams de Thesprotie à la fin de la Seconde Guerre mondiale ou les réfugiés de la guerre civile grecque en direction de Europe de l'Est et de l'URSS ont marqué le siècle, contribuant à remodeler en profondeur la région. De loin le plus massif, l'échange forcé de population entre la Grèce et la Turquie a entraîné l'arrivée sur le territoire de l'État grec de plus d'un million deux cent mille réfugiés dans un pays comptant alors cinq millions d'habitants, bouleversant en profondeur l'organisation sociale du pays ${ }^{36}$.

Tenant compte de ces évènements passés, les migrations contemporaines ont montré la propension des individus à mobiliser l'ensemble des réseaux qui s'offrent à eux dans le cadre d'un projet migratoire. Réseaux familiaux, villageois, communautaires ont ainsi été largement étudiés, de même que l'usage des réseaux de communication et des nouvelles technologies, analysé dans les travaux de Dana Diminescu dans les années $2000^{37}$. De même, Pierre Sintès a montré l'importance, dans les Balkans, de la mobilisation de réseaux préexistants dans les parcours migratoires d'Albanais se rendant en Grèce dans les années 1990. Ces réseaux transfrontaliers, témoins des évènements et mobilités passés mis en sommeil pendant la période socialiste, se sont trouvés réactivés par des hellénophones du sud de l'Albanie pour jouer un rôle important dans leurs parcours migratoires ${ }^{38}$. De la même façon, l'émigration des hellénophones de Macédoine et la remobilisation de liens communautaires anciens ont contribué à orienter certains projets de la coopération transfrontalière européenne entre la Grèce et la Macédoine du Nord ${ }^{39}$. 


\section{Contrôle, nation, circulation}

11 Cette attention portée au temps long permet de mettre en perspective les mobilités contemporaines qui touchent la région. Si les Balkans ont récemment été fortement médiatisés en raison de l'arrivée massive de migrants en provenance du Moyen-Orient, des processus régionaux plus discrets, renvoyant à des pratiques culturellement ancrées, telles que le pečalbarstvo, continuent de se déployer. Cela permet de comprendre en quoi la «crise migratoire ${ }^{40}$ » de 2015-2016 marquait une forme de nouveauté alors que les pays des Balkans se trouvaient pris entre les injonctions de l'UE appelant au contrôle des frontières et le sentiment de n'être, finalement, que peu concernés par des mobilités dont leurs territoires ne semblaient pas être la destination finale. Pour autant, ces injonctions au contrôle des frontières ont surtout rendu explicitement visible la place désormais occupée par les Balkans dans le dispositif de contrôle migratoire européen. Elles sont, de plus, intimement liées aux régimes de circulation des citoyens des pays de la région en direction de l'UE : leur alignement sur l'acquis Schengen et le renforcement du contrôle et de la dimension filtrante des frontières, dans un but de lutte contre les "migrations irrégulières ", ont notamment permis à la Serbie, au Monténégro, à la Macédoine du Nord (2009) puis à l'Albanie et à la Bosnie-Herzégovine (2010) de voir leurs citoyens exemptés de visas de court séjour pour se rendre dans l'espace Schengen.

Ce dernier point illustre la complexification croissante des mouvements migratoires au sein de la péninsule balkanique et l'émergence, dans la région, de différents régimes de circulation en relation avec une politique migratoire qui trie les individus en fonction du cadre administratif dans lequel s'inscrit leur déplacement. Car si l'acteur européen est ici omniprésent en tant que principal prescripteur des règles du contrôle frontalier, les États de la région continuent en partie à conduire une politique nationale concernant le franchissement de leur propre frontière. Ainsi, un État comme la Grèce a$\mathrm{t}$-il introduit un statut administratif particulier, l'omogeneia, pour les personnes qu'il considérait comme Grecs de l'étranger. Renforcé au début des années 1990 pour les omogeneis d'ex-URSS et d'Albanie, ce statut permet de pénétrer librement sur le territoire grec et d'y travailler ${ }^{41}$. Cet exemple met en évidence la pluralité des dynamiques du contrôle migratoire, entre règlements européens et influences liées à des processus de construction nationale ${ }^{42}$. Il illustre également une forme de hiérarchisation des individus en fonction de leur appartenance supposée à une communauté nationale, permettant de leur donner accès à des régimes de circulation plus ou moins libéraux entre leur pays d'origine et le territoire grec. Cet exemple particulier ne constitue d'ailleurs pas une exception. En tant que membre de l'UE, la Bulgarie use de son côté du potentiel de mobilité induit par son passeport à des fins de politique extérieure. En permettant un accès facilité à sa citoyenneté à des Macédoniens attirés par ce document de voyage ${ }^{43}$, elle soutient la diffusion d'un discours officiel qui considère les Macédoniens comme étant en réalité $e^{44}$ des Bulgares. En s'appuyant sur une procédure impliquant que les requérants macédoniens déclarent "se sentir Bulgares", la Bulgarie cherche à diffuser sa vision de l'identité macédonienne, notamment auprès des autorités de Macédoine du Nord. Elle s'oppose par exemple actuellement à l'ouverture des négociations d'adhésion à l'UE de la Macédoine du Nord, conditionnant cette ouverture au rapprochement des autorités 
macédoniennes avec les positions officielles bulgares sur plusieurs questions. Cela impliquerait, entre autres, de reconnaître l'existence d'une minorité bulgare en Macédoine du Nord, minorité principalement constituée d'individus naturalisés depuis le début des années $2000^{45}$. De leur côté, de nombreux Macédoniens profitent de cette politique pour se procurer un passeport leur permettant un accès facilité à la mobilité et aux marchés du travail de l'UE, et l'utilisent dans le cadre de leur projet migratoire.

\section{Les circulations au prisme des Balkans}

On pourrait ici légitimement s'interroger sur la pertinence d'aborder cette question des circulations dans le seul espace balkanique. Poser, entre autres, les relations entre dynamiques de l'ethnicité et circulation pourrait en effet sembler présupposer encore une fois une particularité régionale indépassable. Il n'en est rien. Car les déplacements forcés de populations ou l'accès privilégié à la mobilité de certaines catégories de personnes en fonction de critères ethniques ou nationaux ne sont nullement l'apanage des États de la péninsule balkanique. L'intérêt est ici autre: malgré leurs différences, les cas présentés dans ce dossier concernent des États ayant en commun un passé ottoman plus ou moins lointain, avant que différents processus de fragmentation ne fassent diverger leurs trajectoires. La brève histoire des circulations présentée dans cette introduction souligne de plus les nombreux liens qui les unissent au regard des mobilités au cours des deux siècles précédents, de la pečalba à la "route des Balkans » en passant par les migrations de travailleurs balkaniques vers la Grèce. En considérant le chevauchement des temporalités qui, souvent, se superposent au cours d'une même interaction sociale ${ }^{46}$, c'est une forme d'héritage commun qui nous permet de traiter cette question sur un temps long et d'enrichir ${ }^{47}$, par la diversité des cas, la réflexion sur la place des circulations dans les sociétés contemporaines. À cet égard, les migrations et mobilités récentes qui traversent les Balkans peuvent apparaître à la fois comme les révélateurs et les catalyseurs de reconfigurations des échelles de pouvoir dans les Balkans, et au-delà. Vecteurs de tensions et de crispations, mais aussi de formes de solidarité et de résistance, les circulations nous aident à comprendre les nouvelles formes de pouvoir dans leurs différentes acceptions ainsi que les forces alternatives, les processus de fragmentation, de pluralisation, de redistribution ou de contestation.

\section{Les contributions}

14 Issues de l'anthropologie et de l'histoire, les contributions regroupées dans ce dossier abordent des espaces, des thèmes et des époques divers pour envisager ces dynamiques régionales dans leur complexité. Circulations des personnes, des biens, des idées ou des pratiques sont analysées et mises en regard des évolutions sociopolitiques pour aborder, par les Balkans, un champ de réalités polymorphes.

À partir de l'étude d'expériences migratoires de femmes ukrainiennes travaillant en Grèce, Kira Kaurinkoski pose la question de l'inscription de ces mobilités particulières dans le cadre de la globalisation. Conséquences de l'effondrement de l'URSS, ces migrations se sont progressivement renforcées au fil des années 1990 en direction des pays voisins de l'Ukraine mais également de pays de l'Europe méditerranéenne, et notamment de la Grèce. Si, avant 1991, cette migration concernait surtout des réfugiés politiques grecs ayant quitté le pays après la Seconde Guerre mondiale, sa base sociale 
s'est élargie dans les années 1990 avec des individus se rendant en Grèce munis d'un visa touristique et demeurant sur place après l'expiration de celui-ci. Entre 1998 et 2007, les premiers programmes de naturalisation grecs ont contribué à ce que ces migrants ukrainiens tentent de régulariser leur situation. Et, même si les nouvelles arrivées sont peu nombreuses depuis la crise économique de 2008, les Ukrainiens constituent toujours aujourd'hui une communauté étrangère importante dans le pays. Cette migration revêt cependant des traits spécifiques: sur les 20000 citoyens ukrainiens estimés en Grèce en 2019, 90 \% seraient des femmes, souvent originaires de l'ouest de l'Ukraine. C'est le parcours de certaines d'entre elles que Kira Kaurinkoski examine dans son texte pour montrer comment leur émigration s'inscrit dans la continuité de pratiques de mobilité forgées dans les années 1990, en réponse à la crise économique qui a suivi la chute de l'URSS. En rappelant les motivations principalement économiques, et les circulations financières qui accompagnent ces mobilités, l'auteure traite de la place des travailleuses ukrainiennes dans la société grecque. Elle présente alors cette émigration comme une histoire de dévalorisation et d'exclusion sociale en témoignant des difficiles conditions de vie, de travail et des discriminations vécues. Souvent associées, dans les années 1990, à l'image de la femme prostituée, elles sont aujourd'hui perçues en Grèce comme des employées fiables dans les secteurs des services à la personne ou de l'hôtellerie. Mais, en considérant à la fois cette forme de spécialisation et leur catégorisation en tant qu' « Autres » ethniques (allogeneis) dans la société grecque, l'auteure interroge le lien entre circulation, exclusion et formation d'une communauté sur des bases nationales en migration. Les conséquences de ces déplacements sont également analysées au prisme de la société d'origine, alors que la circulation des personnes, des informations, de l'argent et des idées produit de l'hétérogénéité sociale et économique sur les deux versants de cette migration. Vecteurs de la diffusion d'un modèle économique fondé sur la consommation de masse, ces femmes présentées comme "servantes de la globalisation» en deviendraient également ses agents.

La question des rapports entre population migrante et société d'accueil est également au cœur du texte d'Eleni Sideri. En étudiant la diffusion des modèles administratifs et économiques au regard de pratiques muséographiques, elle questionne la construction, dans une société donnée, d'un discours muséal mettant en avant certains éléments de son passé tout en en laissant d'autres de côté, car ils seraient perçus comme moins conformes. Eleni Sideri prend pour cela l'exemple du musée municipal de la ville grecque de Volos, né des programmes de décentralisation progressive impulsés par l'UE. Ces programmes ont indirectement contribué à l'émergence d'institutions muséales locales et à la production de discours localisés. Elle analyse ensuite les rapports de pouvoir mis en place à Volos autour de la création de ce musée, en étudiant le discours programmatique de l'institution et sa réalisation concrète, en lien avec les autorités locales. Ce programme, qui visait initialement une relecture plus inclusive de l'histoire de la ville conformément aux orientations des politiques culturelles diffusées à l'échelle européenne, semble ici mis en tension par les contingences de la vie politique locale, pour un musée dont l'ouverture a été soumise aux aléas de l'alternance démocratique. Les reconfigurations des rapports de pouvoir sont ici abordées au prisme des migrations, question d'importance pour la ville de Volos. Celle-ci s'est en effet fortement développée suite à l'arrivée des réfugiés d'Asie Mineure en 1923 et elle accueille depuis les années 1990 de nombreux migrants venus d'autres pays des Balkans et d'Europe de l'Est. Plus récemment, comme dans de nombreuses villes de Grèce, des 
arrivants venus du Moyen-Orient, d'Afrique ou d'Asie se sont également installés sur place. En étudiant la première exposition du musée, Eleni Sideri montre comment les rapports de pouvoir locaux ont contribué à la production d'une exposition faisant la part belle aux processus migratoires des populations les plus intégrées au récit national grec et à l'histoire locale, laissant totalement de côté d'autres groupes malgré les travaux d'enquête réalisés par les universitaires associés au projet. Mettant l'accent sur des populations venues d'Asie Mineure dans les années 1920 et sur d'autres groupes à l'histoire bien documentée et ancrée dans le local, les premières expositions présentaient le parcours des populations migrantes dans leurs dimensions locales et en conformité avec le discours national. Enfin, la présentation dans le discours muséal de la résilience de ces groupes, de leur intégration sociale et de leur participation à l'histoire locale conduit Eleni Sideri à replacer les travaux de ce musée dans un contexte muséographique plus large, donnant à voir une version idéalisée de la migration. Elle en vient à lier ces dynamiques locales à des idées et des modèles qui lui semblent circuler à l'échelle européenne.

17 Poursuivant le questionnement sur la conformabilité des dynamiques migratoires, Elif Becan documente l'apparition du label "evlad-ı fatihan» au sein d'associations regroupant des personnes ayant quitté les Balkans pour s'installer en Turquie. Cette dynamique est tout d'abord présentée dans sa dimension historique: en retraçant l'histoire des migrations venues des Balkans en Turquie depuis le début du $\mathrm{xx}^{\mathrm{e}}$ siècle, l'auteure rappelle comment la religion musulmane était alors le critère principal permettant aux migrants d'obtenir le statut de «rentrant au pays » et de bénéficier ainsi d'un ensemble d'aides gouvernementales. Les non-musulmans étaient en revanche considérés comme des résidents provisoires. Remontant le fil de l'histoire, l'auteure rappelle les conditions de l'émergence de cette catégorie à partir du XIII siècle et le contexte de sa réémergence au XIX ${ }^{e}$ siècle. Ce faisant, Elif Becan montre que son utilisation contemporaine vise à revendiquer une identité turque et musulmane et à mettre l'accent sur les sacrifices consentis pour la préserver. La revendication de cette référence historique, qui renvoie à l'installation de populations anatoliennes en Roumélie, à l'islam et à l'identité turque, permet à ces personnes de relier directement leur expérience et leur histoire aux grands récits historiques nationaux diffusés en Turquie. En contextualisant l'appropriation de la catégorie d'evlad-ı fatihan par les associations des grandes villes, elle montre comment cette appellation renvoie également à une revendication de conformité permettant à ces groupes de renforcer leur légitimité au sein d'espaces urbains tels qu'Istanbul, Ankara ou Bursa. Se référant à Eric Hobsbawm ${ }^{48}$, l'auteure montre que le label evlad-ı fatihan correspond bien à une tradition inventée liée à un ensemble de pratiques et de représentations visant à démontrer la conformité d'un groupe avec les normes de l'identité nationale turque. La circulation, génératrice de diversité, est ici réinterprétée a posteriori par l'élaboration d'un discours visant à décrire un particularisme comme étant bien conforme au discours dominant.

18 À l'inverse, l'article d'Alexandra Mourgou aborde de façon directe la circulation comme génératrice de changement. À partir de l'étude des quartiers de réfugiés d'Asie Mineure à proximité du port du Pirée, elle étudie l'émergence de nouvelles pratiques sociales et culturelles consécutives à l'installation de réfugiés. Dans ces espaces qui ont accueilli des migrants venus tout d'abord du reste de la Grèce, puis de très nombreux réfugiés d'Asie Mineure, l'auteure traite des interactions entre les pratiques culturelles de ces 
habitants, les conditions de leur installation et leur vie quotidienne. Les pratiques culturelles de la vie passée imprègnent la précarité du quotidien. Ce sont ces interactions qui ont contribué, d'après l'auteure, à l'émergence du rébétiko. Forgé dans ce contexte d'hybridité, il est présenté dans ce texte comme un "assemblage de pratiques sociales et d'expériences communes». Cette étude permet alors de comprendre comment une telle pratique culturelle s'est développée dans la rencontre entre la mémoire et le patrimoine musical de réfugiés d'Asie Mineure avec une population déjà présente sur place. Tous partageaient l'expérience de conditions de vie difficiles et de marginalité sociale. Cette dernière est également étudiée en détail par l'auteure en accordant une attention particulière aux lieux de l'émergence d'un rébétiko à la fois pratique musicale et univers aux nombreuses caractéristiques non musicales. Alexandra Mourgou montre la façon dont ces lieux ont été d'emblée associés à des espaces de déviance et de criminalité, et replace l'émergence de cette pratique culturelle dans le contexte politique de l'entre-deux-guerres où les représentations nationalistes d'une identité grecque européenne étaient promues de façon exclusive par le pouvoir. Il apparaît ici que la diversité née de la circulation et la reconfiguration des pratiques culturelles qui en a découlé peuvent être mesurées au regard des contradictions et des nuances apportées à un discours national se voulant homogénéisant.

Cette question de l'homogénéisation est aussi centrale dans l'article de Milena Pavlović. Prenant pour cadre le combinat minier de Trepča/Trepça, au Kosovo, l'auteure étudie les relations de pouvoir entre les travailleurs de la mine et les institutions dont ils dépendent. En se basant sur des enquêtes ethnographiques, elle prend le parti d'aborder ces travailleurs à partir de trois générations pour analyser leur perception du partage de l'espace de la mine et leurs rapports avec la surface. Cette division permet d'aborder le fonctionnement de la mine de façon chronologique : la première période est celle de l'autogestion dans la Yougoslavie socialiste ; la seconde, des années 1990 au début de la décennie 2000, est la période de crises, conflits et discontinuités politiques ; et la troisième, celle du Kosovo indépendant. Elle montre ainsi que le rapport à l'espace de la mine et aux autres mineurs est intrinsèquement lié au régime politicoéconomique qui régit le fonctionnement du combinat. Ainsi, la définition du partage chez les ouvriers ayant connu l'autogestion socialiste est-elle différente de celle des générations suivantes. Essentiellement fondée sur une solidarité ouvrière, elle relègue, dans la mine, les considérations nationales à l'arrière-plan. Pour des générations dont l'expérience s'est forgée durant les crises ou après l'indépendance, les biais et les inégalités existant entre dirigeants et ouvriers sont perçus au regard des questions ethno-nationales calquées sur les discriminations déjà existantes, et sur les circulations des travailleurs internes à la Yougoslavie. Alors que le Kosovo apparaît, dès la période yougoslave, comme le cadre propice à l'exercice de l'autonomie pour les populations albanophones, les travailleurs serbes ou monténégrins, souvent qualifiés, ne restent que peu de temps et quittent le Kosovo. Revenant au monde de la mine, Milena Pavlović montre comment les conflits ethno-nationaux qui s'amplifient à partir de la fin des années 1980 obligent chacun à se positionner et contribuent à la division progressive des travailleurs de la mine. Alors que les évènements survenus au Kosovo depuis la fin des années 1990 ont conduit à des déplacements massifs, la gestion des différentes mines du combinat sur des bases ethno-nationales en fonction de leur localisation se présente bien comme le reflet des divisions du pays. Enfin, Milena Pavlović conduit cette analyse en considérant le modèle de gestion économique du combinat. De 
l'autogestion à la privatisation, elle aborde la circulation des modèles de gestion économique dans le cadre de la "transition postsocialiste » pour éclairer l'expérience des différentes générations de travailleurs. À partir de celles-ci, elle montre également comment les récits s'ancrent dans le présent tout en mobilisant dans les discours des éléments du passé, éléments qui mettent en lumière les réinterprétations d'une mémoire de la mine au regard du contexte social et politique du Kosovo.

Ce contexte industriel est aussi celui qu'explore le travail de Juliette Ronsin, qui étudie l'évolution des pratiques spatiales d'immigrés yougoslaves de leur lieu d'origine à l'espace de l'agglomération de Sochaux-Montbéliard, puis au sein de celle-ci. Clôturant le dossier, elle montre comment la circulation des personnes est, dans un premier temps, orientée autour de l'industrie et des activités de l'entreprise Peugeot. C'est dans le cadre d'accords conclus entre l'office national de l'immigration français et les autorités yougoslaves que se déroulent le recrutement puis l'arrivée en France de ces travailleurs yougoslaves. Sur place, l'organisation de l'espace et les mobilités sont ensuite essentiellement orientées vers les activités industrielles et régies par l'entreprise. Cette dernière s'occupe tant de l'hébergement que du transport, ce qui entraîne, de la part des travailleurs immigrés yougoslaves, la mise en place de stratégies d'échappement temporaire dans lesquelles la nature occupe une place centrale : les excursions dans les campagnes environnant l'espace urbain, ou les retours au village en Yougoslavie puis en ex-Yougoslavie apportent des respirations à une vie quotidienne presqu'intégralement orientée vers le travail à l'usine. La circulation est donc ici présentée à la fois comme une ressource via la question des migrations internationales yougoslaves, puis comme un moyen d'échapper, en France, à un quotidien entièrement rythmé par le travail. À ces déplacements des hommes s'ajoute, dans ce travail, une dimension tenant à la diffusion d'une forme d'idéal chez les migrants yougoslaves, ici abordée par l'évolution des trajectoires résidentielles, des hôtels Peugeot à l'accession à la propriété et à l'habitat pavillonnaire.

Partant de ces études de cas issues des Balkans mais marquées par une grande diversité tant dans leurs thématiques que dans leurs cadres spatio-temporels, ce dossier entend contribuer à une réflexion plus large sur les circulations.

\section{NOTES}

1. Selon l'Organisation des Nations unies (ONU), plus de 13 millions de Syriens ont dû quitter leur domicile et plus de 6,5 millions d'entre eux se sont rendus à l'étranger. Source : « Syrie : 10 ans de guerre en 10 chiffres", Onu Info, 15 mars 2021, en ligne: https://news.un.org/fr/story/ 2021/03/1091792 (consulté en juin 2021). À l'heure de conclure ce chapitre introductif, je souhaite exprimer mes remerciements à toutes les personnes qui ont participé à la réalisation de ce dossier. Je remercie tout particulièrement les auteures pour leur engagement et les nombreux échanges qui ont enrichi nos réflexions.

2. DUJMOvic Morgane, SINTÈs Pierre, « Chauvinisme frontalier sur la "route des Balkans" ", Hommes et migrations, $\mathrm{n}^{\circ}$ 1317-1318, 2017, p. 107-115. 
3. JAVOUREZ Guillaume, Permanence et changement aux frontières de l'Europe. Une approche des recompositions frontalières entre Grèce et République de Macédoine, thèse de doctorat, Aix-Marseille Université, 2017.

4. PILLANT Laurence, «Les conséquences socio-spatiales des nouvelles modalités du contrôle migratoire à la frontière gréco-turque ", L'espace politique, vol. 25, $\mathrm{n}^{\circ} 1,2015$, en ligne : https:// doi.org/10.4000/espacepolitique.3369 (consulté en septembre 2021).

5. DANIş Didem, PEROUSE Jean-François, «La politique migratoire turque: vers une normalisation? » Migrations Société, vol. 17, n 98 (Turquie, modernité et migrations), 2005, p. 93-106. 6. BATHAIE Azita, «La Grèce, une étape cruciale dans le parcours migratoire des Afghans depuis la frontière iranienne jusqu'en Europe ", Méditerranée, revue géographique des pays méditerranéens, $\mathrm{n}^{\circ}$ 113, 2009, p. 71-77; PÉROUSE Jean-François, «Migrations, circulations et mobilités internationales à Istanbul ", Dossier de l'IFEA. Migration et mobilités internationales: la plateforme turque, $\mathrm{n}^{\circ} 13,2002$, p. 9-29.

7. «Pays de transit » est une catégorie qui émerge dans les années 1990 et qui renvoie aux pays constituant des étapes le long d'un parcours migratoire dont la destination finale se situerait en Europe de l'Ouest ou du Nord. BACON Lucie et al., «Bulgarie, pays de transit? Arrestations, refoulements et confinements à l'est de l'Union européenne ", dans Annalisa Lendaro (dir.), La crise de l'accueil. Frontières, droits, résistances, Paris, La Découverte, 2019, p. 119-141.

8. DUJMOVIC Morgane, "Le contrôle migratoire en Croatie ", e-Migrinter, $\mathrm{n}^{\circ} 14,2016$, en ligne : https://doi.org/10.4000/e-migrinter.738 (consulté en septembre 2021).

9. GotTMANN Jean, La politique des États et leur géographie, Paris, Armand Colin, 1952.

10. Sur la question des réfugiés, voir notamment : HEIMERL Daniela, « La politique de l'Allemagne à l'égard des réfugiés ex-yougoslaves", Hommes et Migrations, vol.1235, $\mathrm{n}^{\circ} 1,2002$, p. 28-39; MOROKVASIC-MULLER Mirjana, "La guerre et les réfugiés dans l'ex-Yougoslavie », Revue européenne des migrations internationales, vol. 8, n², 1992, p. 5-25; SAMARY Catherine, "Refugiés de l'exYougoslavie face à la forteresse Europe ", Innovation: The European Journal of Social Science Research, vol. 7, nº 2, 1994, p. 189-198. À propos de la guerre de Bosnie, voir BOUGAREL Xavier, Bosnie, anatomie d'un conflit, Paris, La Découverte, 1996.

11. Dans les faits, des mobilités transfrontalières destinées à la réalisation d'achats dans d'autres pays du pacte de Varsovie ou en Allemagne et en Autriche existaient déjà durant la période socialiste, et ce depuis les années 1960-1970. Leur ampleur était cependant sans commune mesure avec celle des phénomènes observés suite aux bouleversements du début des années 1990.

12. MOROKVASIC Mirjana, "Une migration pendulaire, les Polonais en Allemagne ", Hommes et Migrations, $\mathrm{n}^{\circ} 1155$, 1992, p. 31-37; DE TINGUY Anne, «Le phénomène des Tchelnoki et la constitution d'espaces transfrontaliers dans la région de la mer Noire», dans Luyazid Kichou, Méditerranée et Mer Noire entre mondialisation et régionalisation, Paris, L'Harmattan, 2000, p. 471-481; DE TINGUY Anne, «L'élargissement à l'est de l'Union, un nouveau défi pour l'Union européenne », Hommes et Migrations, vol. 1230, nº 1 (Europe, ouvertures à l'est), 2001, p. 5-19.

13. WHITOL DE WENDEN Catherine, "Un essai de typologie des nouvelles mobilités », Hommes et Migrations, $\mathrm{n}^{\circ}$ 1233, 2001, p. 5-12; WILLIAMS Allan, BALÁ Vladimir, «Mobilité internationale en Europe centrale: touristes, commerçants et migrants", Revue européenne des migrations internationales, vol. 18, $\mathrm{n}^{\circ} 1$ (Tourisme et migrations), 2002, p. 37-65.

14. WEBER Serge, Nouvelle Europe, nouvelles migrations : frontières, intégration, mondialisation, Paris, Éditions du Félin, 2007, p. 118.

15. DE TINGUY, «L'élargissement à l'est de l'Union, un nouveau défi pour l'Union européenne », art. cité. 
16. MICHALON Bénédicte, «La périphérie négociée. Pratiques quotidiennes et jeux d'acteurs autour des mobilités transfrontalières entre la Roumanie et la Moldavie ", L'espace politique, $n^{\circ} 2,2007$, en ligne : https://doi.org/10.4000/espacepolitique.902 (consulté en septembre 2021).

17. RAGARU Nadège, «Imaginaires et itinéraires migratoires bulgares en Europe. Une introduction", Balkanologie. Revue d'études pluridisciplinaires, vol.11, n 1-2, 2008, en ligne: https://doi.org/10.4000/balkanologie.873 (consulté en septembre 2021).

18. MOROKVASIC Mirjana, "Migrations et diasporas: les Balkans mobiles ", Balkanologie. Revue d'études pluridisciplinaires, vol. 7, n 1, 2003, en ligne: https://doi.org/10.4000/balkanologie.464 (consulté en septembre 2021); RAGARU Nadège (dir.), Dossier "Partir, revenir: imaginaires migratoires bulgares en Europe », Balkanologie. Revue d'études pluridisciplinaires, vol. 11, n 1-2, 2008, en ligne : https://doi.org/10.4000/balkanologie.813 (consulté en septembre 2021).

19. À titre d'exemple, on notait en 2015 près de 3 millions d'entrées de personnes résidant en Macédoine du Nord sur le territoire grec. Les personnes résidant en Macédoine du Nord représentaient alors le plus gros contingent de ces entrées sur le territoire grec, tous pays d'origine confondus. Voir JAVOUREZ, Permanence et changement aux frontières de l'Europe, op. cit.

20. MOROKVASIC-MULLER Mirjana, «Balkans : les exclus de l'élargissement », Hommes et Migrations, vol. 1230, $\mathrm{n}^{\circ} 1$ (Europe, ouvertures à l'est), 2001, p. 81-93.

21. SINTÈs Pierre, La raison du mouvement. Territoires et réseaux de migrants albanais en Grèce, Paris, Karthala, 2010.

22. Suite notamment à la fin de la dictature des colonels en 1974 et à l'entrée du pays dans ce qui était alors la Communauté économique européenne en 1981. MOROKVAŠIC Mirjana, « Migrations et diasporas", art. cité.

23. MOROKVASIC-MULLER, Balkans : les exclus de l'élargissement, op. cit.

24. SINTÈs, La raison du mouvement, op. cit.

25. SINTÈs Pierre, «La migration des Albanais en Grèce. Difficultés méthodologiques pour une étude géographique ", Revue européenne des migrations internationales, vol. 17, n 3, 2001, p. 67-85.

26. KAURINKOSKI Kira, Le "retour» des Grecs de Russie : identités, mémoires, trajectoires, Athènes, École française d'Athènes, 2020.

27. Si les Albanais représentaient $57 \%$ de l'ensemble des migrants recensés en Grèce en 2001, les Bulgares en constituaient $5 \%$ et les Roumains environ $3 \%$. Les chiffres officiels doivent être considérés comme sous-évalués au regard des très nombreuses mobilités provisoires destinées à l'exercice d'une activité professionnelle saisonnière et informelle. SINTÈs Pierre, « Les travailleurs balkaniques en Grèce ", L'espace géographique, tome 36, n 4, 2007 p. 353-365 ; ANGELIDOU Aliki, "Migrations in the "Neighborhood": Negotiations of Identities and Representations about "Greece" and "Europe" among Bulgarian Migrants in Athens", Balkanologie. Revue d'études pluridisciplinaires, vol. 11, $\mathrm{n}^{\circ} 1-2$ (Partir, revenir: imaginaires et itinéraires migratoires bulgares en Europe), 2008, en ligne : https://doi.org/10.4000/balkanologie.1152 (consulté en septembre 2021); GABĂRSKI Nikolaj, "Les expériences migratoires bulgares en Grèce depuis 1989 ", Balkanologie. Revue d'études pluridisciplinaires, vol.11, $\mathrm{n}^{\circ} 1-2$, 2008, en ligne: https://doi.org/10.4000/ balkanologie.1132 (consulté en septembre 2021).

28. KING Russell, ORUC Nermin, «Editorial Introduction: Migration in the Western Balkans Trends and Challenges ", Journal of Balkan and Near Eastern Studies, vol. 21, n 1, 2019, p. 1-10.

29. вовIC Mirjana, « Forced Migrants in Serbia: Refugees and Internally Displaced Persons - Facts and Figures, Coping Strategies, Future ", Refuge, vol. 26, n 1, 2009, p. 101-110.

30. GREEN Sarah, Notes from the Balkans, Princeton, Princeton University Press, 2005, p. 148.

31. GOUNARIS Basil, «Emigration from Macedonia in the Early Twentieth Century », Journal of Modern Greek Studies, $\mathrm{n}^{\circ}$ 7, 1989, p. 133-153.

32. HARNEY Robert, «A Note on Sources in Urban and Immigrant History ", Canadian Ethnic Studies, vol. $9, \mathrm{n}^{\circ} 2,1977$, p. 60-76. 
33. BROWN Keith, « From the Balkans to Baghdad (via Baltimore): Labor Migrations and the Route of Empire », Slavic Review, vol. 69, n 4, p. 816-834.

34. HRISTOv Petko, «Mobilités du travail (gurbet), stratégies sociales et familiales : une étude de cas dans les Balkans centraux» Balkanologie. Revue d'études pluridisciplinaires, vol. 11, n 1-2, 2008, en ligne : https://doi.org/10.4000/balkanologie.912 (consulté en septembre 2021).

35. BIELENIN-LENCZOWSKA Karolina, «Different Models of Labour Migration in Contemporary Macedonia - or What Does Pečalba Mean Today? ", Ethnologia Balkanica, n 14, 2010, p. 11-26.

36. À l'inverse, 350000 musulmans de Grèce étaient déplacés de Grèce en Turquie.

37. DIMINESCu Dana, «Manifeste : le migrant connecté », Migrations/Société, vol. 17, $\mathrm{n}^{\circ}$ 102, 2007, p. 275-292.

38. SINTÈs Pierre, «Les Albanais en Grèce. Le rôle des réseaux préexistants ", Balkanologie. Revue d'études pluridisciplinaires, vol. 7, $\mathrm{n}^{\circ}$ 1, 2003, p. 111-133.

39. JAVOUREZ Guillaume et al., "Les frontières de la Grèce : un laboratoire pour l'Europe?", L'espace politique, vol. $3, \mathrm{n}^{\circ} 33$ (Frontières de guerre, frontières de paix: nouvelles explorations des espaces et temporalités des conflits), 2017, en ligne : https://doi.org/10.4000/espacepolitique.4432 (consulté en septembre 2021).

40. Malgré son inscription dans des dynamiques plus anciennes, voir DUJMOvic, SINTÈs, "Chauvinisme frontalier sur la "route des Balkans" », art. cité.

41. TSITSELIKIS Konstantinos, «Le droit de la différence : minorités et étrangers en Grèce », dans Pierre Sintès, Gilles De Rapper (dir.), Nommer et classer dans les Balkans, Athènes, École française d'Athènes, 2008, p. 107-121.

42. JAVOUREZ Guillaume, SINTÈs Pierre, «Les migrations balkaniques des années 1990 et 2000 à l'ombre des constructions nationales", Migrations/Société, vol.1, nº 75 (Soixante ans de "libre circulation » en Europe), 2019, p. 81-93.

43. RISTESKI Ljupčo, « Bulgarian Passports - Possibilities for Greater Mobility of Macedonians and/ or Strategies for Identity Manipulation? », Etnoantropozum, nº 10, 2014, p. 81-103.

44. MARINOV Tchavdar, L'impasse du passé: la construction de l'identité nationale macédonienne et le conflit politico-historiographique entre la Bulgarie et la Macédoine, thèse de doctorat, EHESS, Paris, 2006.

45. Voir l'article de Tchavdar Marinov dans ce numéro.

46. GILBERT Andrew et al., "Reconsidering Postsocialism from the Margins of Europe", Anthropology News, vol. 48, n 8, 2008, p. 10-11.

47. BLONDEL et al., "Avant-propos ", Revue d'études comparatives Est-Ouest, vol. 46, nº 4 (Habiter l'espace post-yougoslave), 2015, p. 7-34.

48. HOBSBAWM Eric J., RANGER Terence (dir.), The Invention of Tradition, Cambridge, Cambridge University Press, 1983, p. 2.

\section{AUTEUR}

\section{GUILLAUME JAVOUREZ}

LOTERR, MSH Lorraine, Université de Lorraine guillaume.javourez[at]univ-lorraine.fr 